\title{
Protective effects of daphnetin on sodium taurocholate-induced severe acute pancreatitis in rats
}

\author{
ZHI-YONG LIU ${ }^{1}$, JIAO LIU ${ }^{2}$, KAI-LIANG ZHAO ${ }^{1}$, LI-KUN WANG ${ }^{1,3}$, QIAO SHI ${ }^{1}$, \\ TENG ZUO ${ }^{1}$, TIAN-YI LIU ${ }^{1}$, LIANG ZHAO ${ }^{1}$ and WEI-XING WANG ${ }^{1}$ \\ Departments of ${ }^{1}$ General Surgery and ${ }^{2}$ Critical Care Medicine, Renmin Hospital of Wuhan University, Wuhan, Hubei 430060; \\ ${ }^{3}$ Department of Infectious Diseases, Linyi People's Hospital, Linyi, Shandong 276000, P.R. China
}

Received August 17, 2013; Accepted February 11, 2014

DOI: $10.3892 / \mathrm{mmr} .2014 .1995$

\begin{abstract}
Severe acute pancreatitis (SAP) is the sudden onset of pancreatic inflammation, which is characterized by edema, acinar cell necrosis, hemorrhage and severe inflammation of the pancreas and is associated with a high mortality rate. Daphnetin has been shown to alleviate organ injury in a variety of preclinical animal models of coagulation disorders. The aim of the present study was to investigate the protective effects of daphnetin on severe acute pancreatitis in a rat model. Severe acute pancreatitis in the rat model was induced by retrograde infusion of $5 \%$ sodium taurocholate $(1 \mathrm{ml} / \mathrm{kg})$ into the bile-pancreatic duct. Daphnetin $(4 \mathrm{mg} / \mathrm{kg}$ ) was administered intraperitoneally at $30 \mathrm{~min}$ prior to the infusion of sodium taurocholate. The severity of pancreatitis was evaluated by various analyses of serum amylase and lipase, tumor necrosis factor- $\alpha$ (TNF- $\alpha)$ and interleukin-1 $\beta$ (IL-1 $\beta$ ) levels, myeloperoxidase (MPO) activity and malondialdehyde (MDA) content, as well as by histological grading. The levels of TNF- $\alpha$ and IL- $1 \beta$ in the serum were measured by ELISA. The results revealed that the daphnetintreated SAP rat group (SAP-D) exhibited a lower pathological score of the pancreas compared with the SAP group (SAP). Further analyses demonstrated that the SAP-D group had lower levels of serum amylase, lipase and pro-inflammatory cytokines, including TNF- $\alpha$ and IL-1 $\beta$, and a decreased MPO activity and MDA content 3, 6 and $12 \mathrm{~h}$ subsequent to the infusion of sodium taurocholate compared with the SAP group (SAP). These findings indicated that daphnetin exerted a protective function in the SAP rat model. Therefore, daphnetin may be considered as
\end{abstract}

Correspondence to: Professor Wei-Xing Wang, Department of General Surgery, Renmin Hospital of Wuhan University, 238 Jiefang Road, Wuhan, Hubei 430060, P.R. China

E-mail: sate.1lite@163.com

Abbreviations: MPO, myeloperoxidase; MDA, malondialdehyde; SAP, severe acute pancreatitis; H\&E, hematoxylin and eosin; TNF- $\alpha$, tumor necrosis factor- $\alpha$; IL- $1 \beta$, interleukin- $1 \beta$

Key words: severe acute pancreatitis, daphnetin, inflammation, cytokine a potential compound for the therapy and prevention of acute pancreatitis.

\section{Introduction}

Acute pancreatitis (AP) is the sudden onset of inflammation of the pancreas. The majority of acute pancreatitis cases are mild, self-limiting and have few or no complications. However, $20-25 \%$ of acute pancreatitis progress to severe acute pancreatitis (SAP), a serious disease associated with a high morbidity rate of $10-20 \%(1,2)$. The mechanisms of AP remain to be fully identified. Activation of digestive enzyme zymogens within pancreatic acinar cells is hypothesized to be a critical initiating event. Once the disease process is initiated, the inflammatory response is invoked and results in local pancreatic injury. If this inflammatory reaction is significant, it leads to a systemic inflammatory response syndrome and subsequent multiple organ dysfunction syndrome $(1,3)$. Previous studies have revealed that pancreatic damage due to SAP leads to the release of pro-inflammatory cytokines (4-6). Among the family of pro-inflammatory cytokines, tumor necrosis factor- $\alpha$ (TNF- $\alpha)$ and interleukin-1 $\beta$ (IL-1 $\beta)$ are believed to be the most significant ones; they have potent properties in terms of enhancing local tissue destruction, inducing distant organ complications and increasing the overall mortality rate of acute pancreatitis (7). Numerous anti-inflammatory cytokine therapies have been tested in SAP animal models and were demonstrated to be effective (8). However, clinical trials of anti-inflammatory cytokine therapies have at present failed to achieve similar effects (9). Thus, further investigation is required to test novel therapeutic approaches.

Dracaena marginata (D. marginata) is a well-known flowering plant. The roots, stems, leaves and flowers of $D$. marginata can be used as medicinal materials, which exert a variety of biological functions, including reducing inflammation, promoting blood circulation, relieving blood stasis and eliminating blood stagnation to relieve pain. The chemical composition of D. marginata mainly includes bisflavones, lignans and coumarins (10). Among these coumarins, daphnetin has been reported to exhibit significant contributions to the analgesic and anti-inflammatory activity of Daphne odora (D. odora). As shown in Fig. 1, daphnetin (7,8-dihydroxycoumarin), an active monomer ingredient extracted from 
D. marginata, has a potent inhibitory effect on serum TNF- $\alpha$ and IL-1 in adjuvant-induced arthritic rats (11), and can be used to treat various autoimmune diseases including rheumatoid arthritis and systemic lupus erythematosus. Despite of the increasing knowledge implicating the involvement of TNF- $\alpha$ and IL-1 in the progression of acute pancreatitis, no animal study or clinical trial directly pertaining to daphnetin treatment of pancreatitis has been reported yet. Therefore, the present study was designed to investigate the protective effects of daphnetin on SAP in a rat model.

\section{Materials and methods}

Reagents. Daphnetin (a monomeric compound) was isolated from D. marginata extract by Tauto Biotech Co., Ltd. (Shanghai, China). The purity of daphnetin used in the present study was $>99.2 \%$. Sodium taurocholate was purchased from Sigma-Aldrich (St. Louis, MO, USA).

Animals. Male Wistar rats (200-250 g) were obtained from the Center of Experimental Animals of Hubei Academy of Medical Sciences in China (Hubei, China). The animals were fed with standard rat chow and tap water ad libitum, and were hosted in plastic cages with controlled temperature $\left(20-22^{\circ} \mathrm{C}\right)$ and humidity (50-52\%). The room was maintained on a $12 \mathrm{~h}$ light-dark cycle throughout the study. All the rats were acclimated for at least one week prior to use. Food was withdrawn $12 \mathrm{~h}$ prior to the experiment. The experiments were performed in accordance with the Guide for the Care and Use of Laboratory Animals (National Research Council of USA, 1996), and it was approved by the local Ethics Committee of Wuhan University (Wuhan, China).

Induction of SAP. Prior to the experiment, rats were deprived of food, but drinking water was available ad libitum. An SAP model was induced as the method described previously (12) with minor modifications. Anesthesia was administered by intraperitoneal injection of $10 \%$ chloraldurat $(3 \mathrm{ml} / \mathrm{kg})$ and rats underwent sterile laparotomy. The bile-pancreatic duct was cannulated through the duodenum and SAP was induced by a standardized pressure-controlled retrograde infusion of $5 \%$ sodium taurocholate $(0.1 \mathrm{ml} / \mathrm{kg}$; Sigma-Aldrich Chemie $\mathrm{GmbH}$, Steinheim, Germany) into the bile-pancreatic duct. Following infusion, the section of the bile-pancreatic duct entering the duodenum was clipped by a non-invasive vascular clip for $5 \mathrm{~min}$. The vascular clip was then removed and the abdomen was closed. The sham-operated group underwent the same procedure as the SAP group but sterile saline was used instead of $5 \%$ sodium taurocholate.

Experimental design. A total of 72 male Wistar rats were randomly divided into four groups, two of which did not recieve treatment (groups 1 and 3) and two (groups 2 and 4) that were treated with daphnetin $(4 \mathrm{mg} / \mathrm{kg})$. Daphnetin was dissolved in saline. Group 1 (SAP group, $n=18$ ): SAP was induced with an injection of $5 \%$ sodium taurocholate into the common biliopancreatic duct, daphnetin was not administered. Group 2 (SAP-D group, $n=18$ ): SAP was induced with an injection of $5 \%$ sodium taurocholate into the common biliopancreatic duct, $4 \mathrm{mg} / \mathrm{kg}$ daphnetin was administered intraperitoneally

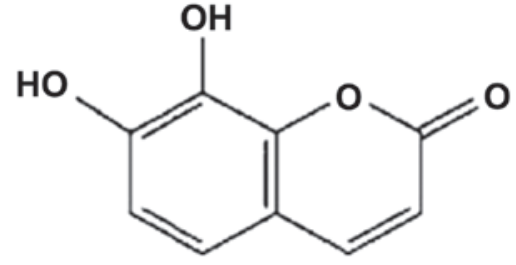

Figure 1. Structure of daphnetin.

30 min prior to sodium taurocholate. Group 3 (SO group, $\mathrm{n}=18)$ : Rats were sham-operated and daphnetin was not administered. Group 4 (SO-D group, n=18): Rats were shamoperated and daphnetin was administered at a dose of $4 \mathrm{mg} / \mathrm{kg}$ intraperitoneally $30 \mathrm{~min}$ prior to modeling. Following surgery, $1 \mathrm{ml}$ saline solution per $100 \mathrm{~g}$ body weight was administered subcutaneously for fluid replacement.

Six rats from each group were randomly sacrificed under chloraldurat anesthesia at respective time points $(3,6$, and $12 \mathrm{~h})$ subsequent to the induction of pancreatitis or sham-surgery. Rats underwent thoracotomy by midsternal incision and $>3 \mathrm{ml}$ blood was obtained from the left ventricle of the heart. Blood samples were centrifuged at 3,000 x g for $15 \mathrm{~min}$, and serum was stored at $-20^{\circ} \mathrm{C}$ until further analysis. The heads of the pancreas were harvested and fixed with $40 \mathrm{~g} / 1$ formaldehyde for histological analyses. The rest of the pancreatic tissues were rinsed with saline, blotted dry, snap frozen in liquid nitrogen and stored at $-80^{\circ} \mathrm{C}$ for further use.

Histopathological analysis. Sections of the paraffin-embedded tissues were prepared for pathological analysis with hematoxylin and eosin (H\&E) staining. Morphometric documentation for pancreatic sections under light microscopy (Olympus Optical Ltd., Tokyo, Japan) was evaluated by two independent pathologists who were blinded to the study. The sections of pancreatic tissue were scored for the severity of pancreatitis based on edema, inflammation, vacuolization and necrosis according to the scale described by Schmidt et al (13).

Measurement of serum amylase and lipase. Amylase and lipase levels were measured using standard techniques with a Full Automatic Chemistry analyzer (Olympus AU 2700 Analyzer, Olympus Inc., Tokyo, Japan).

Measurement of tissue MPO and MDA. Myeloperoxidase (MPO) activity was determined by a spectrophotometric method using a commercial assay kit according to the manufacturer's instructions (Nanjing Jiancheng Bioengineering Institute, Nanjing, China). The absorbance was tested at a wavelength of $460 \mathrm{~nm}$ during a period of $5 \mathrm{~min}$. The results were expressed as units of enzyme activity of MPO per gram of wet tissue (U/g wet tissue).

The pancreatic malondialdehyde (MDA) content was measured to evaluate the severity of pancreatic peroxidation injury by a thiobarbituric acid colorimetric method using an MDA assay kit (Nanjing Jiancheng Corp., Nanjing, China). The harvested pancreas tissue was homogenized with $10 \%$ (w/v) of $20 \mathrm{mmol} / \mathrm{l}$ potassium phosphate buffer $(\mathrm{pH} 7.4)$ containing $30 \mathrm{mmol} / \mathrm{K} \mathrm{KCl}$ and then centrifuged at $1500 \mathrm{x} \mathrm{g}$ for $15 \mathrm{~min}$. The MDA concentration was calculated from the standard curve and presented as nmol/mg. 
A

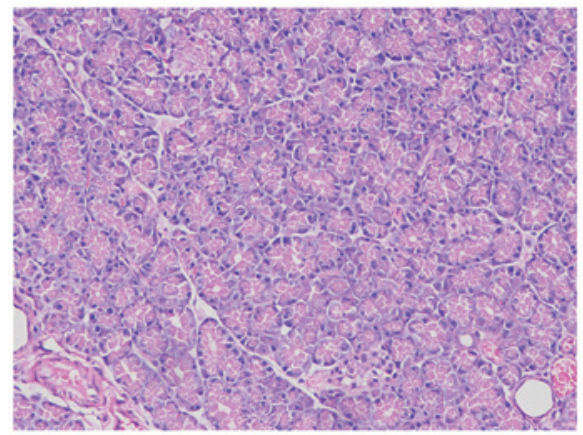

C

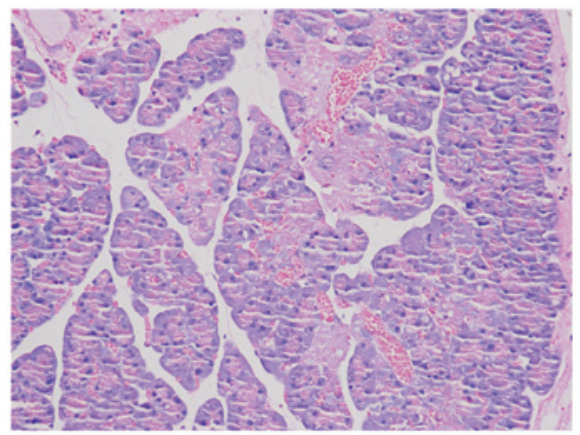

B

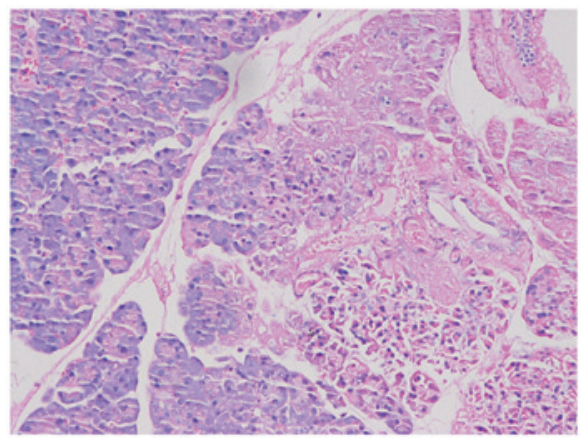

D

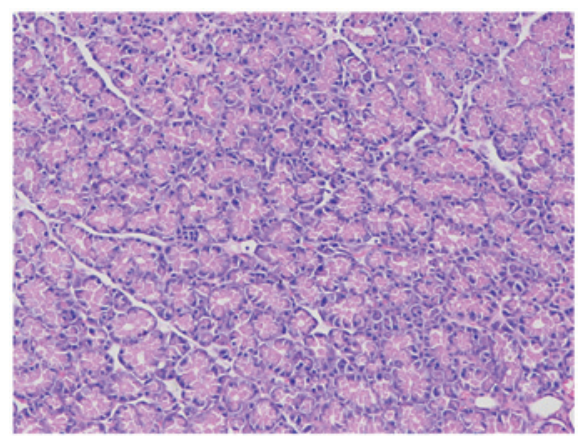

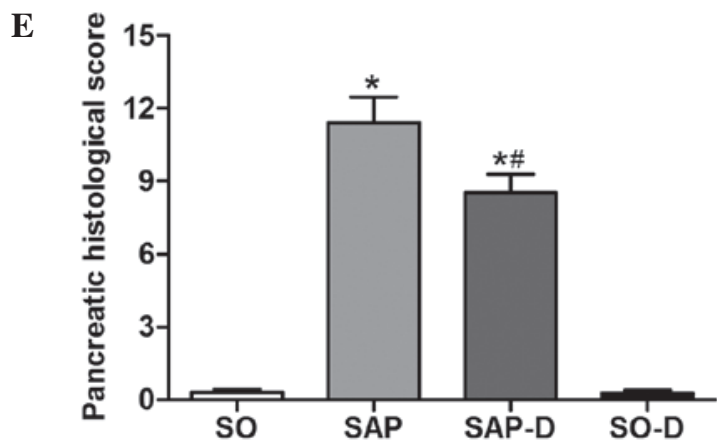

Figure 2. Representative hematoxylin and eosin staining sections of the pancreas. (A) SO group at $12 \mathrm{~h}$. (B) SAP group at $12 \mathrm{~h}$. (C) SAP-D group at $12 \mathrm{~h}$. (D) SO-D group at $12 \mathrm{~h}$. (E) Comparison of the total pathological score of the pancreas. SO, sham-operated control group; SAP, sodium taurocholate group; SAP-D, sodium taurocholate + daphnetin group; SO-D, sham-operated + daphnetin group. "P $<0.01$ vs. the SO group; "P<0.05 vs. the SAP group (original magnification, x200). SAP, severe acute pancreatitis.

$T N F-\alpha$ and IL-1 $\beta$ determination. Serum concentrations of TNF- $\alpha$ and IL-1 $\beta$ were determined by the commercially available enzyme-linked immunosorbent assay (ELISA), according to the manufacturer's instructions (R\&D Systems, Minneapolis, MN, USA). The absorbance was read on an automated microplate reader (Thermo Multiskan MK3; Thermo Fisher Scientific Oy, Vantaa, Finland) and the concentrations were calculated according to the standard curve run on each assay plate. All the samples were assayed in duplicate.

Statistical analysis. The data are expressed as the mean \pm standard deviation. Following comparison of the homogeneity of variance (homogeneity of variance test), one-way analysis of variance was used to determine whether there were any significant differences among the means of different treatment groups, followed by the Student-Newman-Keuls multiple comparison test to determine the difference between two groups within the multiple groups demonstrating significant differences. All statistical analyses were performed by statistical software (SPSS Base 17.0 for Windows, SPSS Inc., Chicago, IL, USA). P $<0.05$ was considered to indicate a statistically significant difference.

\section{Results}

Pretreatment with daphnetin reduces inflammatory changes in the pancreas. Example images of the pancreas tissue stained with H\&E are shown in Fig. 2A-E. Sodium taurocholate-induced pancreatic damage was verified by increased edema, inflammatory cell infiltration, vacuolization and necrosis. Pretreatment with daphnetin reduced the inflammatory changes in the pancreas. The pathological scores of the pancreas injury in the SAP and SAP-D groups were significantly increased at $12 \mathrm{~h}$ compared with the $\mathrm{SO}$ and SO-D groups $(\mathrm{P}<0.01)$. In the SAP-D group, the total score of pancreas injury was significantly lower compared with the SAP group at $12 \mathrm{~h}(\mathrm{P}<0.05)$.

Effects of daphnetin on serum amylase and lipase. As shown in Fig. 3, baselines of serum amylase and lipase were detected in the SO group. Compared with baseline levels, serum amylase and lipase levels were not significantly altered in the SO-D group, but were significantly increased in the SAP group at each time point $(\mathrm{P}<0.01)$. Peak serum amylase and 

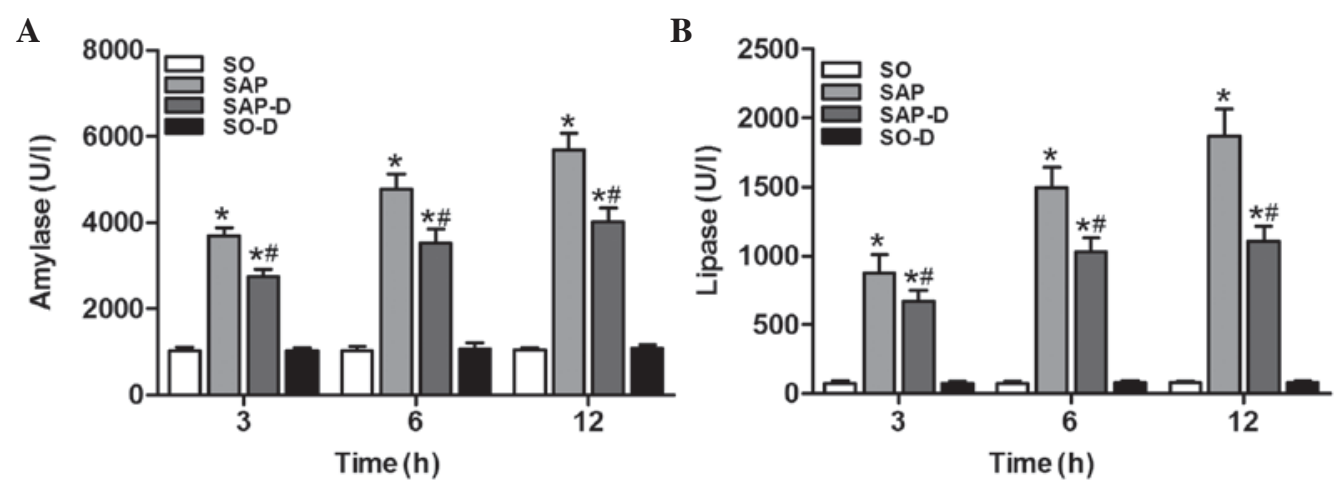

Figure 3. Levels of (A) serum amylase and (B) lipase. SO, sham-operated group as the control group; SAP, sodium taurocholate group; SAP-D, sodium taurocholate + daphnetin group; SO-D, sham-operated + daphnetin group. ${ }^{*} \mathrm{P}<0.01$ vs. the SO group at the same time point; ${ }^{*} \mathrm{P}<0.05$ vs. the SAP group at the same time point. SAP, severe acute pancreatitis.
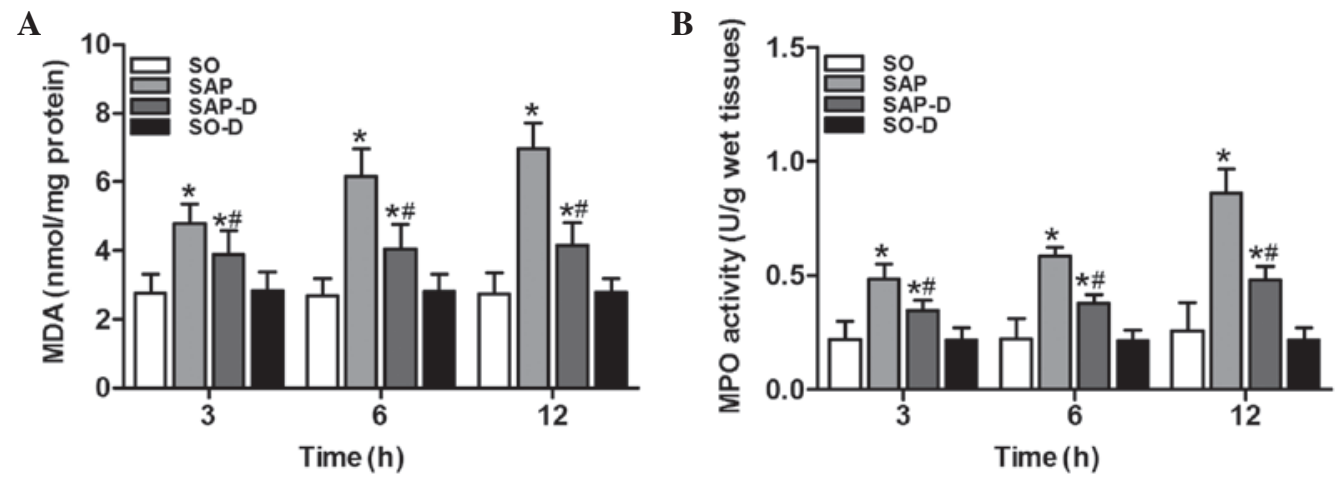

Figure 4. Pancreatic tissue (A) MDA content and (B) MPO activity. SO, sham-operated control group; SAP, sodium taurocholate group; SAP-D, sodium taurocholate + daphnetin group; SO-D, sham-operated + daphnetin group. " $\mathrm{P}<0.01$ vs. the $\mathrm{SO}$ group at the same time point; " $\mathrm{P}<0.05$ vs. the SAP group at the same time point. MDA, malondialdehyde; MPO, myeloperoxidase; SAP, severe acute pancreatitis.
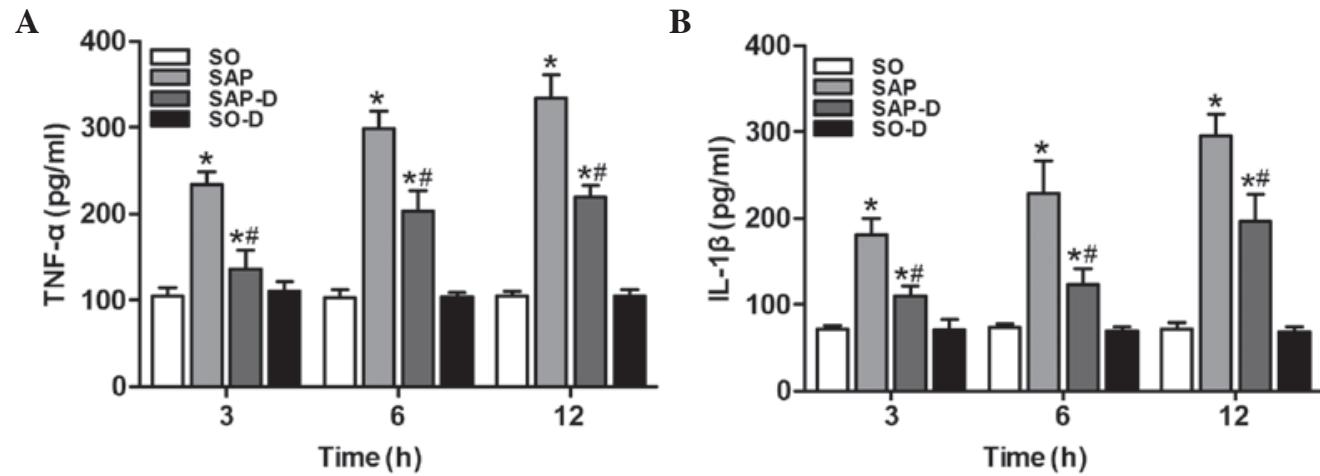

Figure 5. Effects of daphnetin inhibition on pro-inflammatory cytokine production. Serum levels of TNF- $\alpha$ and IL-1 $\beta$ were quantified by ELISA. Results are expressed as the mean \pm standard error of the mean. SO, sham-operated group as the control group; SAP, sodium taurocholate group; SAP-D, sodium taurocholate + daphnetin group; SO-D, sham-operated + daphnetin group. " $\mathrm{P}<0.05$ vs. the SO group at the same time point; " $\mathrm{P}<0.05$ vs. the SAP group at the same time point. TNF- $\alpha$, tumor necrosis factor- $\alpha$; IL-1 $\beta$, interleukin- $1 \beta$; SAP, severe acute pancreatitis.

lipase levels were observed at $12 \mathrm{~h}$ in the SAP group, but were significantly reduced at 3,6 and $12 \mathrm{~h}$ in the SAP-D group, compared with the SAP group $(\mathrm{P}<0.05)$.

Daphnetin significantly decreases MDA content and MPO activity. The MDA content and MPO activity of pancreatic tissue in the SAP group was significantly increased at each time point compared with the SO group and SO-D group
$(\mathrm{P}<0.01)$, but there were no significant differences between the SO group and SO-D group. Daphnetin significantly decreased the MDA content and MPO activity of pancreatic tissue at each time point in the SAP-D group compared with the SAP group ( $\mathrm{P}<0.05$; Fig. 4$)$.

Effects of daphnetin inhibition on pro-inflammatory cytokine production. The serum levels of TNF- $\alpha$ in the SAP 
group were significantly higher compared with that in the SO group $(\mathrm{P}<0.01)$. However, the serum level of TNF- $\alpha$ in the SAP-D group subsequent to treatment with daphnetin (4 mg/kg) was significantly lower compared with that in the SAP group at each time point $(\mathrm{P}<0.05)$. There was no significant difference between the SO-D and SO groups (Fig. 5A).

A significant difference was identified in the serum IL-1 $\beta$ levels between the SAP group and $\mathrm{SO}$ group $(\mathrm{P}<0.01)$. However, there was no significant difference between the SO-D and SO groups. Following treatment with daphnetin $(4 \mathrm{mg} / \mathrm{kg})$, the serum IL-1 $\beta$ in SAP-D group was significantly lower compared with that in the SAP group at each time point $(\mathrm{P}<0.05$; Fig. 5B).

\section{Discussion}

Daphnetin, regarded as the active principal for quality control of Zushima-Pian, has been used in China as an oral treatment for rheumatoid arthritis $(14,15)$. Several studies have shown that daphnetin is involved in analgesic and anti-inflammatory activity, exerts antidiarrheal, antihypoxic, antiparasitic and larvicidal properties, inhibits protease activity and produces therapeutic effects in collagen-induced arthritis (16-19). A previous study also demonstrated that daphnetin exhibited a potent inhibitory effect on the serum TNF- $\alpha$ and IL-1 levels in adjuvant-induced arthritic rats (11). In the present study, a sodium taurocholate-induced pancreatitic rat model was used to assess the protective effect of daphnetin on SAP.

SAP characterized by edema, acinar cell necrosis, hemorrhage and severe inflammation of the pancreas, is a common disease in which the underlying mechanisms of the local initiating events in the pancreas have not been fully clarified. However, the inflammatory mediator theory proposed previously provides a basis for further investigation (20). In the early stage of AP the excessively activated pancreatic enzymes damage adjacent pancreatic cells and lead to local infiltration of inflammatory cells including macrophages and neutrophils. These cells and pancreatic cells produce and release significant pro-inflammatory cytokines. It was shown in the present study that among various inflammatory mediators correlated with AP, TNF- $\alpha$ and IL- $1 \beta$ are currently hypothesized to be the most significant in terms of enhancing local tissue destruction, producing distant organ complications and increasing the overall mortality rate of acute pancreatitis (7). TNF- $\alpha$ and IL-1 $\beta$ are known to mediate tissue injury by upregulating the levels of adhesion molecules, producing nitric oxide, activating inflammatory cells to release other cytokines and oxygen free radicals, which result in lipid peroxidation, cellular swelling and cell death, thus these are significant parameters that indicate the severity of AP (21). In the present study, daphnetin was found to exhibit an inhibitory effect on serum TNF- $\alpha$ and IL-1 $\beta$, and reduced the severity of sodium taurocholateinduced necrotic pancreatitis without side effects.

At present, the standard treatment of AP remains based on supportive care, the search for novel therapies that alter the natural history of the disease is a continuing challenge for a number of researchers. Emerging evidence indicates that inflammatory cytokines are promising therapeutic targets for improving the course of the disease. Several agents have been tested in an attempt to inhibit the effects of inflammatory mediators that have an established role in the pathogenesis of AP (22). Inhibition of TNF- $\alpha$ or IL-1 $\beta$ reduces the local and systemic inflammatory response. These findings provide a potential development in the treatment of AP. Previously, different inhibition therapies have been evaluated in experimental pancreatitis $(23,24)$. The present study provides the evidence that treatment with daphnetin significantly reduces the development of taurocholate-induced pancreatitis in rats, diminishes necrotic areas, pancreatic hemorrhage and inflammation, and lipid peroxidation.

Patients with AP present with elevated blood levels of pancreatic digestive enzymes, including amylase and lipase (20). In the present study, marked elevation of the pancreatic enzymes was observed in the SAP group. However, pretreatment with daphnetin in the SAP-D group significantly reduced serum amylase and lipase levels as compared with the SAP group. In addition, daphnetin significantly decreased the MPO activity and MDA content in pancreatic tissue in the treated groups with taurocholate-induced pancreatitis.

Neutrophils are crucial in the development of AP (25). Their infiltration into inflamed tissue contributes to the inactivation of foreign antigens and to the remodeling of injured tissue; however, exaggerated recruitment into the pancreas accounts for tissue destruction via the production of reactive oxygen metabolites, granule enzymes and cytokines that amplify the inflammatory response (26). In the present study, daphnetin reduced neutrophil infiltration to the inflamed area of the pancreas in daphnetin-treated rats, which represents a significant additional mechanism for its protective anti-inflammatory effects. The possible cause of the ameliorative effect of daphnetin on the histopathological and biochemical findings of AP may be attributed to the prevention of lipid peroxidation, which results in a decrease of the inflammatory reaction and oxygen free inhibition of TNF- $\alpha$ and IL- $1 \beta$ activity.

Daphnetin exerted multiple effects, including inhibiting TNF- $\alpha$ and IL-1 $\beta$ activity, decreasing the levels of amylase and lipase, reducing the secretion of inflammatory cytokines and ameliorating the injury of pancreatic tissue, which resulted in the protection of the rats with SAP. However, these novel observations are based on animal models, and the definite mechanisms of how daphnetin affects AP remain to be fully elucidated. On account of species differences that exist between humans and rats, more emphasis in further studies should be placed on human in vivo studies to elucidate the role of daphnetin in health and disease at different stages of pancreatitis.

In conclusion, administration of daphnetin decreased the severity of sodium taurocholate-induced acute pancreatitis in rats, which was associated with inhibition of the TNF- $\alpha$ and IL-1 $\beta$ activity. Daphnetin exerted a protective effect in the pancreatitis model, and may be a potential novel therapeutic agent for SAP.

\section{References}

1. Renzulli P, Jakob SM, Täuber M, Candinas D and Gloor B: Severe acute pancreatitis: case-oriented discussion of interdisciplinary management. Pancreatology 5: 145-156, 2005.

2. Lund H, Tønnesen $H$, Tønnesen MH and Olsen O: Long-term recurrence and death rates after acute pancreatitis. Scand J Gastroenterol 41: 234-238, 2006

3. Bhatia M, Brady M, Shokuhi S, Christmas S, Neoptolemos JP and Slavin J: Inflammatory mediators in acute pancreatitis. J Pathol 190: 117-125, 2000. 
4. Norman J: The role of cytokines in the pathogenesis of acute pancreatitis. Am J Surg 175: 76-83, 1998.

5. Ogawa M: Acute pancreatitis and cytokines: 'Second attack' by septic complications leads to organ failure. Pancreas 16: 312-315, 1998.

6. Mayer J, Rau B, Gansauge F and Beger HG: Inflammatory mediators in human acute pancreatitis: clinical and pathophysiological implications. Gut 47: 546-552, 2000.

7. Denham W, Yang J, Fink G, Denham D, Carter G, Ward K and Norman J: Gene targeting demonstrates additive detrimental effects of interleukin-1 and tumor necrosis factor during pancreatitis. Gastroenterology 113: 1741-1746, 1997.

8. Norman JG, Fink GW, Messina J, Carter G and Franz MG: Timing of tumor necrosis factor antagonism is critical in determining outcome in murine lethal acute pancreatitis. Surgery 120: 515-521, 1996.

9. Baumgartner JD and Calandra T: Treatment of sepsis: past and future avenues. Drugs 57: 127-132, 1999.

10. Zhang W, Zhang WD, Liu RH, Shen YH, Zhang C, Cheng HS, Fu P and Shan L: Two new chemical constituents from Daphne odora Thunb. var. marginata. Nat Prod Res 20: 1290-1294, 2006.

11. Gao Q, Shan J, Di L, Jiang L and Xu H: Therapeutic effects of daphnetin on adjuvant-induced arthritic rats. J Ethnopharmacol 120: 259-263, 2008.

12. Paszkowski AS, Rau B, Mayer JM, Möller P and Beger HG: Therapeutic application of caspase 1 /interleukin-1beta-converting enzyme inhibitor decreases the death rate in severe acute experimental pancreatitis. Ann Surg 235: 68-76, 2002.

13. Schmidt J, Rattner DW, Lewandrowski K, Compton CC Mandavilli U, Knoefel WT and Warshaw AL: A better model of acute pancreatitis for evaluating therapy. Ann Surg 215: 44-56, 1992.

14. Shen HF, Bao CD and Lu JH: The clinical study of Zushima Tablet in the treatment of rheumatoid arthritis. Chin J Inf Tradit Chin Med 6: 38-39, 1999 (In Chinese).

15. Chen ZL, Li XH and Yang Y: Efficacy analysis on treating rheumatoid arthritis with Zushi Mapian. Clin J Chin Med 3: 64-65, 2011 (In Chinese)

16. Feily A and Reza Fallahi H: Potential utility of daphnetin as a novel treatment for pemphigus vulgaris. G Ital Dermatol Venereol 145: 557-558, 2010.
17. Fylaktakidou KC, Hadjipavlou-Litina DJ, Litinas KE and Nicolaides DN: Natural and synthetic coumarin derivatives with anti-inflammatory/antioxidant activities. Curr Pharm Des 10: 3813-3833, 2004

18. Ye B, Zheng YQ, Wu WH and Zhang J: Iron chelator daphnetin against Pneumocystis carinii in vitro. Chin Med J (Engl) 117: 1704-1708, 2004

19. Tu L, Li S, Fu Y, Yao R, Zhang Z, Yang S, Zeng X and Kuang N: The therapeutic effects of daphnetin in collagen-induced arthritis involve its regulation of Th17 cells. Int Immunopharmacol 13: 417-423, 2012.

20. Hirota M, Nozawa F, Okabe A, Shibata M, Beppu T, Shimada S, Egami H, Yamaguchi Y, Ikei S, Okajima T, Okamoto K and Ogawa M: Relationship between plasma cytokine concentration and multiple organ failure in patient with acute pancreatitis. Pancreas 21: 141-146, 2000.

21. Laveda R, Martinez J, Munoz C, Penalva JC, Saez J, Belda G, Navarro S, Feu F, Mas A, Palazon JM, Sanchez-Paya J, Such J and Perez-Mateo M: Different profile of cytokine synthesis according to the severity of acute pancreatitis. World J Gastroenterol 11: 5309-5313, 2005.

22. De Almeida JL, Jukemura J, Coelho AM, Patzina RA, Machado MC and da Cunha JE: Inhibition of cyclooxygenase-2 in experimental severe acute pancreatitis. Clinics (Sao Paulo) 61: 301-306, 2006.

23. Oruc N, Ozutemiz AO, Yukselen V, Nart D, Celik HA, Yuce G and Batur Y: Infliximab: a new therapeutic agent in acute pancreatitis. Pancreas 28: e1-e8, 2004.

24. Luan ZG, Zhang J, Yin XH, Ma XC and Guo RX: Ethyl pyruvate significantly inhibits tumour necrosis factor- $\alpha$, interleukin- $1 \beta$ and high mobility group box 1 releasing and attenuates sodium taurocholate-induced severe acute pancreatitis associated with acute lung injury. Clin Exp Immunol 172: 417-426, 2013.

25. Genovese T, Mazzon E, Di Paola R, Muià C, Crisafulli C, Menegazzi M, Malleo G, Suzuki H and Cuzzocrea S: Hypericum perforatum attenuates the development of cerulein-induced acute pancreatitis in mice. Shock 25: 161-167, 2006.

26. Cuzzocrea S, Mazzon E, Dugo L, Serraino I, Centorrino T, Ciccolo A, Van de Loo FA, Britti D, Caputi AP and Thiemermann C: Inducible nitric oxide synthase-deficient mice exhibit resistance to the acute pancreatitis induced by cerulein Shock 17: 416-422, 2002. 